\title{
A Behavioral Analysis of Habituation and Sensitization of Shortening in the Semi-Intact Leech
}

\author{
Nicholas M. Boulis and Christie L. Sahley \\ Department of Biology, Yale University, New Haven, Connecticut 06511
}

\begin{abstract}
We have previously demonstrated that the shortening reflex of the leech Hirudo medicinalis displays habituation, dishabituation, and sensitization. In this paper we demonstrate that the shortening reflex of the semi-intact animal also displays these phenomena. In the first experiment we found that the magnitude of the touch-elicited shortening reflex decreased as a result of repeated stimulations of the skin every 2 min. The second experiment examined the change in this reflex as a function of the interstimulus interval (ISI). The reflex failed to decrease when the ISI was $10 \mathrm{sec}$, but decreased significantly when the ISI was either 45 or 360 sec. Finally, in a third experiment we found that the presentation of noxious stimuli prior to habituation training prevented habituation. Thus, as we have observed previously in the intact animal, the semi-intact animal displays habituation, dishabituation, and sensitization.
\end{abstract}

It is becoming clear that the simpler forms of nonassociative learning, habituation, and sensitization may be important building blocks for the expression of the more complex forms of associative learning, classical and instrumental conditioning (Kandel and Schwartz, 1982). For this reason, we have begun our analysis of learning in the annelid Hirudo medicinalis with the study of habituation, dishabituation, and sensitization of the touch-elicited shortening reflex.

The cellular and molecular mechanisms mediating habituation and sensitization have been explored in a number of vertebrate and invertebrate species. Perhaps the most extensive analysis of habituation and sensitization has been done by Kandel and his colleagues (Kandel, 1976, 1977; Kandel and Schwartz, 1982) on the gill- and siphon-withdrawal reflex of the marine mollusk Aplysia californica. This reflex, readily elicited by a light tactile stimulus, shows a dramatic reduction in amplitude as a result of repeated presentations of the stimulus (Pinsker et al., 1970). Habituation, in the short-term form, can last for several minutes to a few hours. Following repeated habituation, training sessions can last for weeks (Carew et al., 1972). The amplitude of the reflex can be restored by delivering a strong sensitizing stimulus to the animal (dishabituation). The same reflex can be enhanced following the presentation of a noxious

\footnotetext{
Received Oct. 23, 1987; revised Feb. 1, 1988; accepted Mar. 2, 1988.

We wish to thank Drs. Mike Davis and Jerry Rudy, and Ms. Tracy Karrer for helpful comments and criticisms of the manuscript. In addition, we would like to thank Drs. Nelson Donnegan, Jerry Rudy, and Don Weisz for numerous discussions of the data. This work was supported by Grant R01MH37902, Whitehall Foundation Grant 56840, and a Sloan Fellowship to C.L.S.

Correspondence should be addressed to Dr. Sahley, Department of Biology, Yale University, Box 6666, New Haven, CT 06511.

Copyright (c) 1988 Society for Neuroscience $0270-6474 / 88 / 124621-07 \$ 02.00 / 0$
}

stimulus (sensitization), and this behavioral modification can be present in either a short- or a long-term form (Pinsker et al., 1973). Habituation is thought to be due largely to homosynaptic depression at the synapses of the siphon sensory cells onto their follower cells (Castellucci et al., 1970; Castellucci and Kandel, 1976). Sensitization appears to result from heterosynaptic facilitation at the same synapses (Byrne et al., 1978; Hawkins et al., 1981). Although less is known regarding the underlying neural events mediating habituation and sensitization in the leech, recent work has concentrated on defining the behavioral parameters of these phenomena. Several examples of habituation have been described in the leech. Gee (1913) first demonstrated that repetitive presentations of a shadow or a jarring motion resulted in progressively lesser disruptions of an ongoing ventilatory response. Kaiser (1954) extended these findings and showed that the rate of habituation to the shadow was negatively correlated to the intensity of the light. Lockery (Lockery et al., 1985; Lockery and Kristan, 1985) has recently demonstrated habituation of the photic-elicited shortening response. Stoller and Sahley (1985) have described habituation, sensitization, and classical conditioning of the touch-elicited shortening response in the intact animal. In addition to modulating the shortening reflex, habituation has been observed to affect more complex behaviors, such as the shock-elicited swimming response (Debski and Friesen, 1985) and the water current-elicited motor response (Ratner, 1972).

In this paper, we examine habituation, dishabituation, and sensitization of the touch-elicited shortening reflex in a semiintact preparation. Our goal is to develop a preparation that allows us to monitor cellular events while simultaneously measuring the behavioral response. We found that the behavior of the semi-intact preparation parallels that of the intact animal (Stoller and Sahley, 1985). This affords us the opportunity to use this preparation to study the cellular events mediating behavioral plasticity. By studying the cellular changes during training, we hope to reveal new mechanisms that may not be apparent when using an end-point analysis in which changes in the nervous system are determined prior to and following training.

\section{Experiment 1}

\section{Habituation and dishabituation in semi-intact leech}

The purpose of Experiment 1 was to determine whether the touch-elicited shortening response of the semi-intact preparation could be modified by habituation and dishabituation. The intact leech shows a significant decrease in responding as a result of 20 repeated tactile stimuli at a 2 min interstimulus interval (ISI) (Stoller and Sahley, 1985). Responsiveness is restored following a shock to the tail. We repeated this training protocol to 


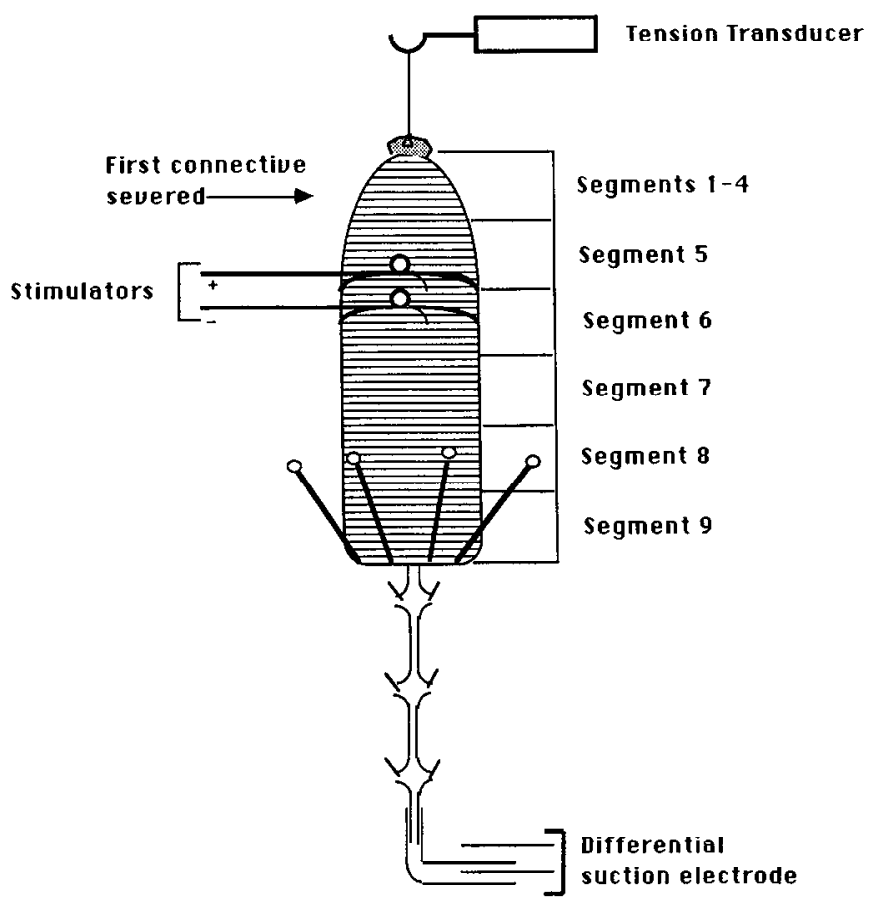

Figure 1. Schematic representation of the semi-intact preparation. The preparation consists of the anterior 9 segments of the leech. The preparation is connected to a tension transducer to measure anterior-posterior contractions. Silver wire electrodes are implanted in the dorsal skin of the leech and are used to deliver the habituating stimulus and the dishabituating stimulus.

determine whether this behavior in the semi-intact preparation was similar to that observed in the intact animal.

\section{Materials and methods}

Animals. Jumbo (1.5 grm) leeches (Hirudo medicinalis) supplied by Ricamparex, Bordeaux, France were used throughout the experiment. About 10-15 animals were housed at room temperature in a $13 \times 18 \times 10.5$ $\mathrm{cm}$ plexiglass container in about $0.5-1$ in. of leech water $(0.5 \mathrm{gm}$ instant ocean/liter of distilled water). The water was changed daily. Since the leeches are shipped from France, they are allowed to acclimate to the lab for at least 1 week prior to experimentation. Likewise, no leeches were used after they had been in the lab for over 3 months.

Semi-intact preparation. In order to simultaneously record hehavior and cellular activity, the following preparation was designed. First, a small incision was made at the animal's fifth annulus on its ventral side. The nerve cord (connective) was severed between the fused ganglia and the first segmental ganglion in order both to eliminate the complicating effects that might be contributed by the fused head ganglia (Kristan et al., 1982) and to incapacitate the anterior sucker.

In order to measure the shortening response, a $20 \mathrm{~cm}$ silk suture thread (Ethicon 6-0) was attached to the anterior sucker and to a force transducer (Bionix Electronics Model EDI la). Last, the leech was transected at the ninth segment, leaving 2 ganglia and the associated sinus exposed. The sinus on the remaining connective caudal to the second ganglion was removed. This preparation was then secured to a Sylgard $(0.5-\mathrm{cm}$-deep) lined Petri dish $(9 \mathrm{~cm}$ in diameter) with 4 stainless steel pins placed at the line of transection. The exposed ganglia were pinned out behind the animal with shortened minuten pins (FST stainless steel; $0.1 \mathrm{~mm}$ in diameter). The nerve connective was sucked into a suction electrode. It was necessary to leave 2 ganglia exposed in order to relieve the stress on the electrode connection. Further, the ganglion stability that is achieved in this fashion is essential to future efforts aimed at intracellular recording. The behavioral response was recorded on a Gould 2-channel recorder (Model 220). A schematic representation of this preparation is presented in Figure 1.

Stimulus materials. The stimulus used to elicit shortening was a shock provided via silver wires attached to a Grass stimulator (Model S88).
Two Teflon-coated silver wires $(0.2 \mathrm{~mm}$ in diameter) were secured around the leech. Approximately $3 \mathrm{~mm}$ of Teflon was removed from each wire and was hooked into the dorsal surface of each leech. These wires were connected through a Grass stimulus-isolation unit (Model SI U5) to a Grass stimulator.

Individual animals were allowed to make a sufficient adjustment to the semi-intact preparation, as indicated by a stable baseline. This adjustment period usually took between 10 and $20 \mathrm{~min}$. The stimulus voltage level was titrated to a magnitude slightly above the threshold for eliciting a response. Such a stimulus facilitated the observation both of increases and decreases in behavior, while causing minimal damage to the preparation. This voltage level was used as the stimulus. All shocks were $3 \mathrm{msec}$ in duration. Following this titration, the leech was again allowed to return to a stable behavioral baseline.

Dependent measure. The dependent measure was the amount of shortening elicited by the conditioned stimulus (CS) recorded as the amplitude of the contraction (in $\mathrm{mm}$ ), as measured by the tension transducer.

Data analysis. The first response was scored as $100 \%$, and all subsequent responses were scored as a percentage of the initial contraction. Each animal's scores were averaged in 5 trial blocks. In addition, 2 difference scores were calculated for each animal: (1) the difference between the first and the last block of habituation training (Block 1 Block 4), and (2) the difference between the last block of habituation training and the block of trials following presentation of the dishabituating stimulus (Block 4 - Block 5). Both types of data were analyzed in 1 of 2 ways. In Experiment 1, because only one experimental group was used, $t$ tests were used. In the remaining experiments, in which multiple experimental groups were employed, an analysis of variance (ANOVA) was used to analyze the performance of the various groups across blocks and Newman-Keuls post hoc tests were used to assess significant ANOVA main effects and interactions (Keppel, 1973).

Training regimen. Eight semi-intact preparations were analyzed in this experiment. Twenty stimuli were presented at a 2 min ITI. Two minutes following the last stimulus presentation, a dishabituating shock was delivered. This consisted of 2 trains of 10 shocks each at a rate of 10 shocks/sec. The dishabituating stimuli were $2 \mathrm{~V}$ larger than the small shock used as the habituating stimulus. The 2 min ITI was maintained for the delivery of the dishabituating shocks. To assess the effect of the dishabituating stimulus, 3 additional test stimuli were presented again at a 2 min ITI.

\section{Results and discussion}

The mean percentages of the initial contractions on habituation Block 1 (H1), Block 4 (H4), and dishabituation Block 1 (D1) are presented in Figure 2 . As shown in Figure $2 A$, the amplitude of the reflex response significantly decreased as a result of the 20 habituation training trials: $t(7)=6.5, p<0.001$. Likewise, following the dishabituating stimulus, there was a significant increase in the response: $t(7)=2.35, p<0.05$. In fact, the dishabituating stimulus almost restored the response to its initial level: $t(7)=1.2, p<0.3$. The difference scores indicated that each animal's response decreased approximately $46.5 \pm 7 \%$ from Block 1 to Block 4 and increased 26.1 $\pm 13 \%$ from Block 4 to 5 . The difference scores are presented in Figure $2 B$.

These results thus demonstrate that a semi-intact leech preparation without the fuscd hcad ganglia can display both habituation and dishabituation much as in the intact animal. Thus, the study of the reflex, and especially its plasticity, can be pursued in the segmental ganglia, which are relatively less complicated than the fused ganglia.

\section{Experiment 2}

\section{Habituation and dishabituation over various ISIs}

Since nothing is known about the properties of the semi-intact leech preparation, the purpose of Experiment 2 was to assess changes in the shortening reflex as a function of a basic parameter, the interstimulus interval. In this experiment, the values of this parameter were investigated: 10, 45, and $300 \mathrm{sec}$. Fol- 
lowing habituation training, the dishabituating stimulus used in Experiment 1 was delivered and the reflex reassessed.

\section{Materials and methods}

The protocol used in Experiment 2 was identical to that of Experiment 1 except that, following the setup of the preparation, each leech was randomly assigned to one of 3 groups. Eight semi-intact preparations were run at each of 3 ITIs, $10 \mathrm{sec}, 45 \mathrm{sec}$, and $5 \mathrm{~min}$. These particular intervals were chosen to bracket the 2 min interval used in Expcriment 1. Following the last stimulus, dishabituating stimuli were delivered to the leech. Since these stimuli were presented twice, the appropriate ITI was maintained for the delivery of the facilitating shocks. Following these stimuli, 3 additional stimuli were presented at the same ITI and voltage level as were used during habituation training. In addition, a fourth group of animals $(n=6)$ was run as a control group to assess the effect of total time in the experiment on the responsiveness of the preparations. Leeches in the fourth group were treated exactly as those in the experimental groups except that, following baseline measurements, the preparations were left undisturbed in their training dishes. After $100 \mathrm{~min}$ (the total time in the dish for leeches in the $5 \mathrm{~min}$ group), tests were conducted exactly as described for the experimental groups.

\section{Results and discussion}

\section{Habituation}

The mean percentages of initial contraction for Block 1 (first 5 trials) and Block 4 (last 5 trials) for leeches in all 3 ITI groups are presented in Figure 3 . The ANOVA revealed a significant group effect: $F(2,18)=4.64, p<0.05$, block effect: $F(1,2)=$ $28.1, p<0.01$; and a significant group $\times$ block interaction: $F(2,18)=10.86, p<0.01$. As seen in Figure 3, there were no differences in the percentages of the initial contractions across the first block among leeches in all 3 groups. However, as revealed by Newman-Keuls post hoc analysis of the significant group $\times$ block interaction, leeches in the $10 \mathrm{sec}$ group showed no difference in responding from Block 1 to Block 4 , whereas leeches in both the 45 and 300 sec groups showed a significant decrease in responding from trial Block 1 to trial Block 4. Moreover, the post hoc analysis also revealed that the $300 \mathrm{sec}$ group showed significantly less responding in the last block of habituation training than did leeches in either of the other 2 training groups (Ncwman-Kculs post hoc analysis; $p<0.05$ ). Since the percentage of initial contractions was used in these analyses, and their normalization procedure could mask differences in absolute scores, a separate ANOVA was run on the absolute initial contractions of the groups. The results of this analysis indicated no significant group effect: $F(2,21)=0.48, p=0.625$. In addition, leeches in the time in dish control group showed no decrease in responding over the $100 \mathrm{~min}$ time interval. The mean of the posttest scores was $111 \pm 17.3 \%$ of the pretest scores. A paired $t$ test (pretest vs posttest) indicated no difference in responding between the 2 tests: $t(5)=0.69, p=0.53$.

Analysis of the difference scores also supports this finding. A 1-way ANOVA of the Block 1 - Block 4 difference scores for leeches in each of the ITI groups revealed a significant group effect: $F(2,18)=9.892, p<0.01$. Newman-Keuls post hoc analysis indicated that there was a significant difference in responding among the 3 groups; animals in the $5 \mathrm{~min}$ group showed the greatest difference between Block 1 and Block 4 performance, while animals in the $10 \mathrm{sec}$ group showed no difference in responding from Block 1 to Block 4.

\section{Dishabituation}

As seen in Figure 4 and revealed by the ANOVA, responding was significantly greater following the dishabituating stimulus; block effect: $F(1,18)=1.02, p<0.01$. Moreover, Newman-
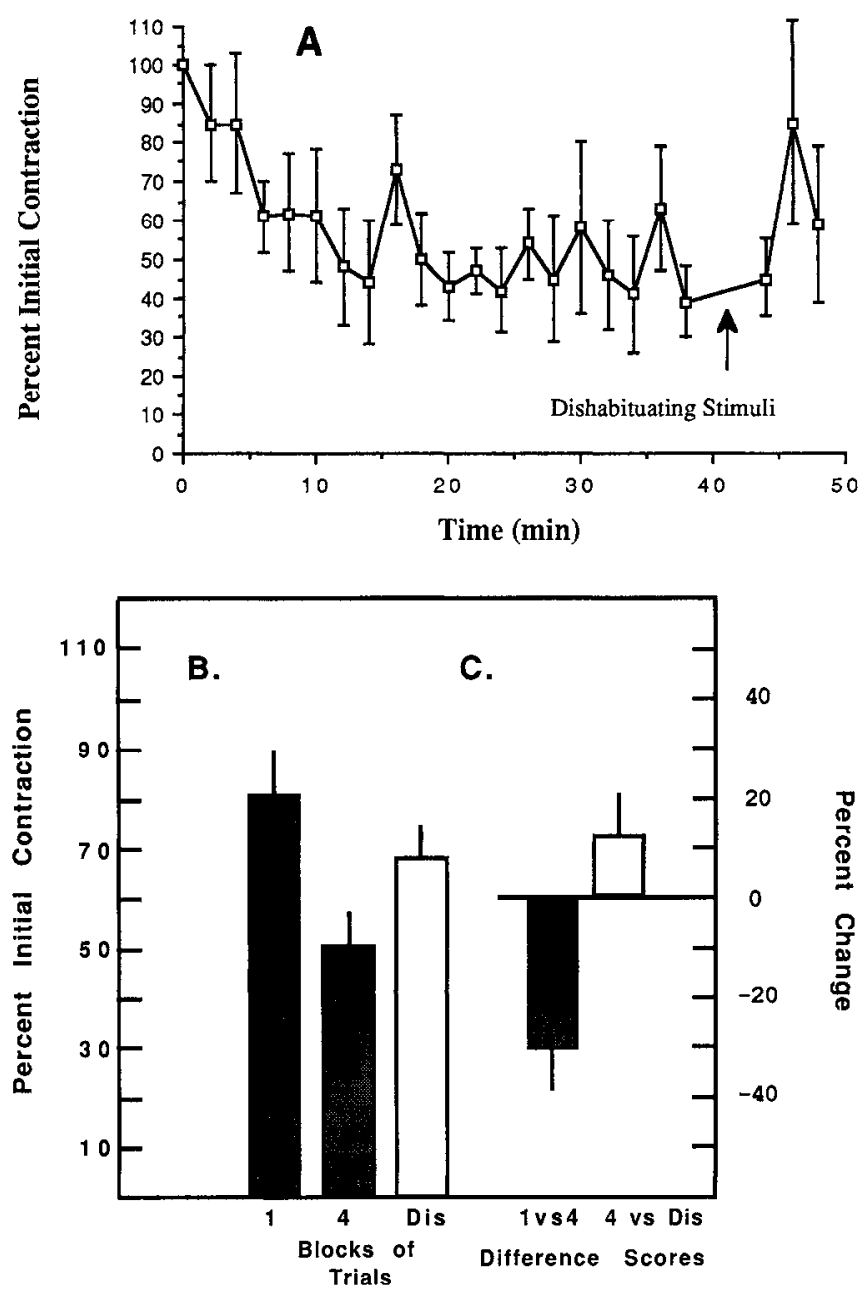

Figure 2. A, A trial-by-trial presentation of the mean percentage initial contraction for Experiment 1 . The arrow represents the delivery of the dishabituating stimuli. $B$, Mean percentage initial contraction for the first block of habituation trials $(1)$, the last block of habituation trials (4), and the block of trials following presentation of the dishabituating stimulus $(D I S)$. $C$, Mean difference scores between the first and the last blocks of training, and between the last block of training and the block of trials following the dishabituating stimulus.

Keuls analysis of the significant group effect $(F(2,18)=20.64$, $p<0.01$ ) indicated that leeches in the $10 \mathrm{sec}$ group responded more overall than leeches in either the 45 or 2 min group. The interaction was not reliable.

This pattern of results is supported by the analysis of the difference scores. Leeches in all ITI groups showed a similar increase in the magnitude of responding from Block 4 to 5; group effect: $F(2,18)=0.674, p<0.5$.

The results of this experiment replicate and extend our findings from Experiment 1. First, repeated presentations of a tactile stimulus result in the habituation of the touch-elicited shortening reflex and the reflex is restored following the presentation of a noxious stimulus. Second, the degree of habituation varies as a function of ITI. That is, although habituation of the shortening reflex is observed at both the $45 \mathrm{sec}$ and $5 \mathrm{~min}$ intervals, it is most dramatic at the $5 \mathrm{~min}$ interval. Moreover, at the 10 sec interval no decrease in responding is observed. The decrement in responding is not due simply to the time in the dish, since preparations held in the dish for $100 \mathrm{~min}$ maintain responding at $85 \%$ of their initial response amplitude. 


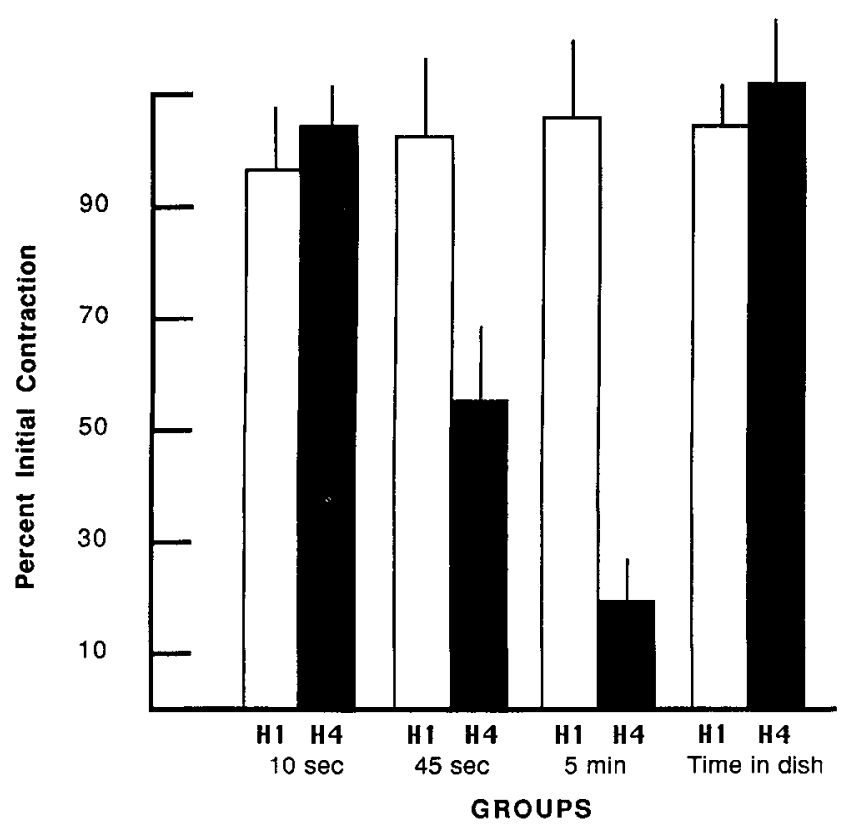

Figure 3. The mean percentage initial contraction for the first block of habituation trials (white bars) and for the last bar of habituation training (black bars) for leeches in the $10 \mathrm{sec}, 45 \mathrm{sec}$, and 5 min groups. No decrement in responding is seen for leeches in either the $10 \mathrm{sec}$ group or the time in dish control group.

This pattern of responding is consistent with several, but not all, aspects of the dual-process theory of habituation (Groves and Thompson, 1970). Briefly, the theory states that any stimulus presented to an animal produces 2 internal responses, a decrementing process and a facilitating process. The interaction of these 2 processes determines the resultant behavioral response to the stimulus. The characteristics of the stimulus are critical in the determination of the resultant behavior. A facilitating process may have a relatively shorter duration and thus dissipate more quickly than the decrementing process. The results of Experiment 2 are consistent with this interpretation. The existence of a facilitatory process with a shorter duration than that of an antagonistic decrementing process would explain the results of Experiment 2 . The observed ITI (group) $\times$ block interaction would occur if, at the shorter intervals, sensitization produced by the stimulus had insufficient time to dissipate. In contrast, the longer ITIs would provide an adequate time interval for sensitization to dissipate and the effect of habituation on the reflex could emerge.

However, the dual-process theory also states that habituation is directly affected by the frequency of stimulus presentation, such that more rapid habituation is observed at shorter ITIs (Groves and Thompson, 1970; Thompson et al., 1973). Our findings are just the opposite; more habituation is scen at longer intervals. Recent reports question the idea of increased habituation at shorter ITIs and suggest that the rapid response decrement observed at short ITIs may be due to motor fatigue and/ or prepulse inhibition and therefore does not reflect greater habituation (Davis, 1970; Ison and Hammond, 1971; File, 1973). It could be that the shortening reflex is difficult to fatigue or that the leech is insensitive to prepulse inhibition, and therefore we are seeing habituation unconfounded by fatigue or prepulse inhibition. The answer will remain unclear until the relevant cellular data are obtained. However, Groves et al. (1969) suggest

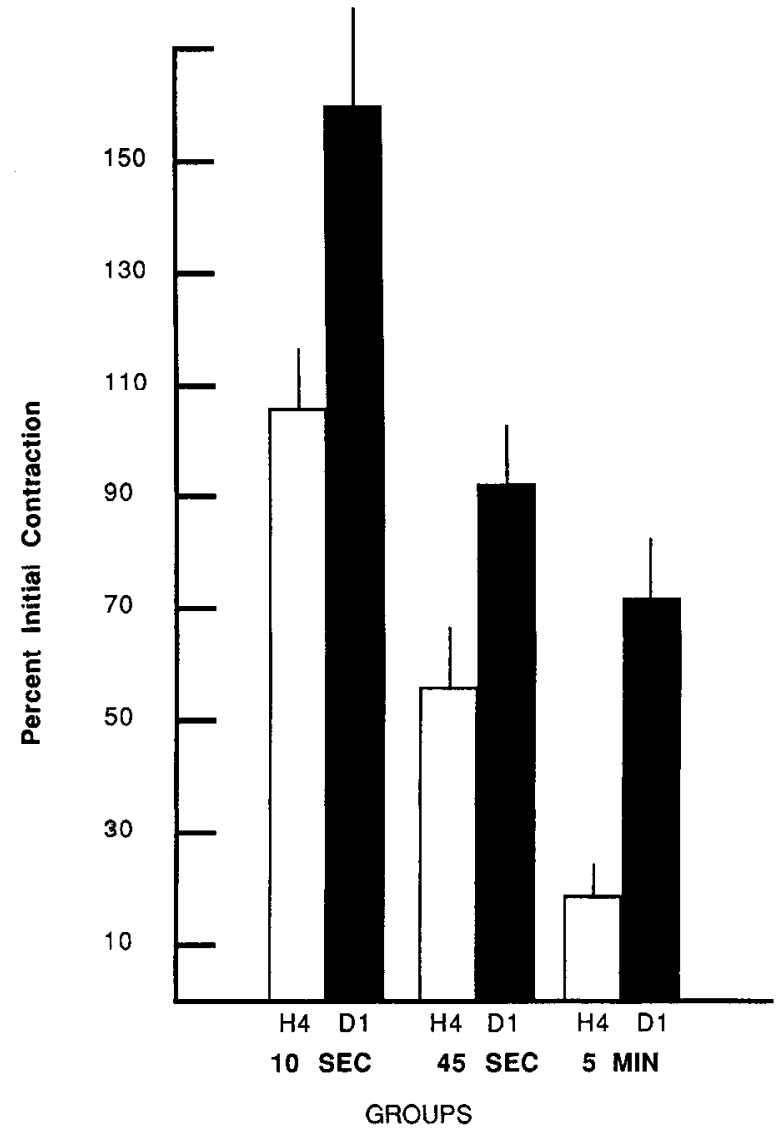

Figure 4. Mean percentage initial responding for the last block of habituation training (white bars) and the block of trials following the dishabituating stimulus (black bars) for leeches in the 3 interval groups. Leeches in all groups show a significant increase in responding following the dishabituating stimulus.

that if a stimulus is capable of causing even short-term transient sensitization, the effects of frequency on habituation are confounded. That is, the final behavioral outcome is critically dependent on both the frequency and intensity of the stimulus and their interaction. The sensitizing effects of a stimulus can be observed early in habituation; responding on the initial trials increases and then decreases as training progresses. We see this pattern of responding in $90 \%$ of the leeches in these studies. Thus we suggest that the critical variable in our studies may be the intensity of the habituating stimulus and that with a very weak stimulus we would observe more rapid habituation at shorter intervals.

As for dishabituation, however, no differences were observed in the magnitude of this response. The difference scores indicated that the same magnitude of facilitation was produced by the dishabituating stimulus regardless of the level of responding produced by the habituation training.

\section{Experiment 3}

Sensitization of the shortening reflex

Sensitization refers to the increased responsiveness in an animal's behavior following an experience with a strong, often noxious, stimulus (Domjan and Burkhardt, 1984). Sensitization has been described on both behavioral and cellular levels for a number of vertebrate and invertebrate species. Perhaps the bestdescribed analysis of sensitization is for the gill- and siphon- 


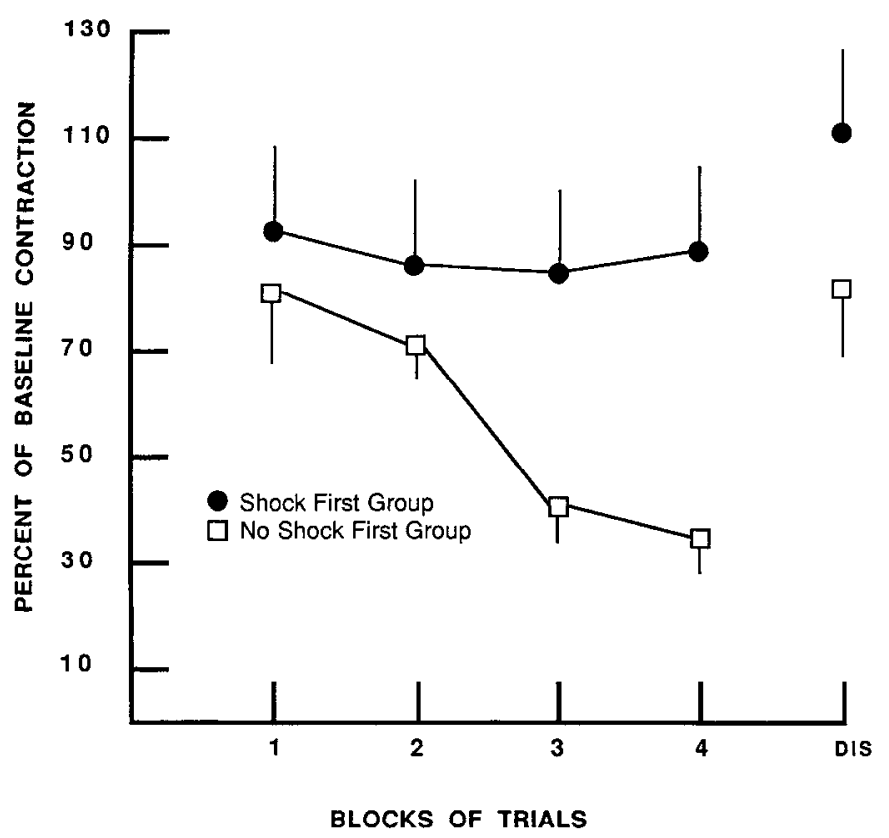

Figure 5. Mean percentage initial contraction for leeches in the shock and no-shock groups across habituation training trials (Blocks $1-4$ ) and following dishabituation ( $D I S$; Block 5$)$. No habituation is seen for leeches in the shock group.

withdrawal reflex of the marine mollusk Aplysia californica (Hawkins et al., 1987). In these experiments, sensitization is reflected as an increase in the magnitude of the gill- or siphonwithdrawal reflex. No data are available on the time course of this sensitization, nor is there evidence regarding the effect of sensitization on subsequent habituation. For this reason we have examined the effect of a sensitizing stimulus both on the level of initial responsiveness of the shortening reflex prior to habituation training and on its effect on the rate of the subsequent habituation.

\section{Materials and methods}

Ten leeches were randomly divided into 2 groups, shock (S) and no shock (NS). The dissection procedures were exactly as described in Experiment 1. The training procedures were also identical to those described in Experiment 1, except that prior to the first stimulus, 2 stimuli were administered to establish a baseline behavioral response. Following the initial baseline test stimulus, the animals in Group $S$ received 2 sensitizing stimuli identical to those used to dishabituate in Experiments 1 and 2. Each of these stimuli was given at an ITI of 2 min and preceded the rest of the stimuli by $2 \mathrm{~min}$. Two minutes following the sensitizing stimulus, regular habituation training with a 2 min ITI began. Two minutes following the last training trial, the dishabituating stimulus was presented.

\section{Results and discussion}

The mean percentages of the initial contractions in 5 trial ulocks for the 2 groups are presented in Figure 5. As is apparent, animals that received the sensitizing treatment showed very little decrement in responding over the habituation training session as compared to animals that did not receive this stimulus. The ANOVA revealed a significant group effect: $F(1,8)=11.58, p$ $<0.01$; block effect: $F(1,8)=5.69, p<0.05$; and group $\times$ block interaction: $F(1,8)=6.35, p<0.05$. The Newman-Keuls post hoc analysis of the interaction indicated that no significant differences were observed between Blocks 1 and 4 for animals in the shock group, while, in contrast, animals in the NS group

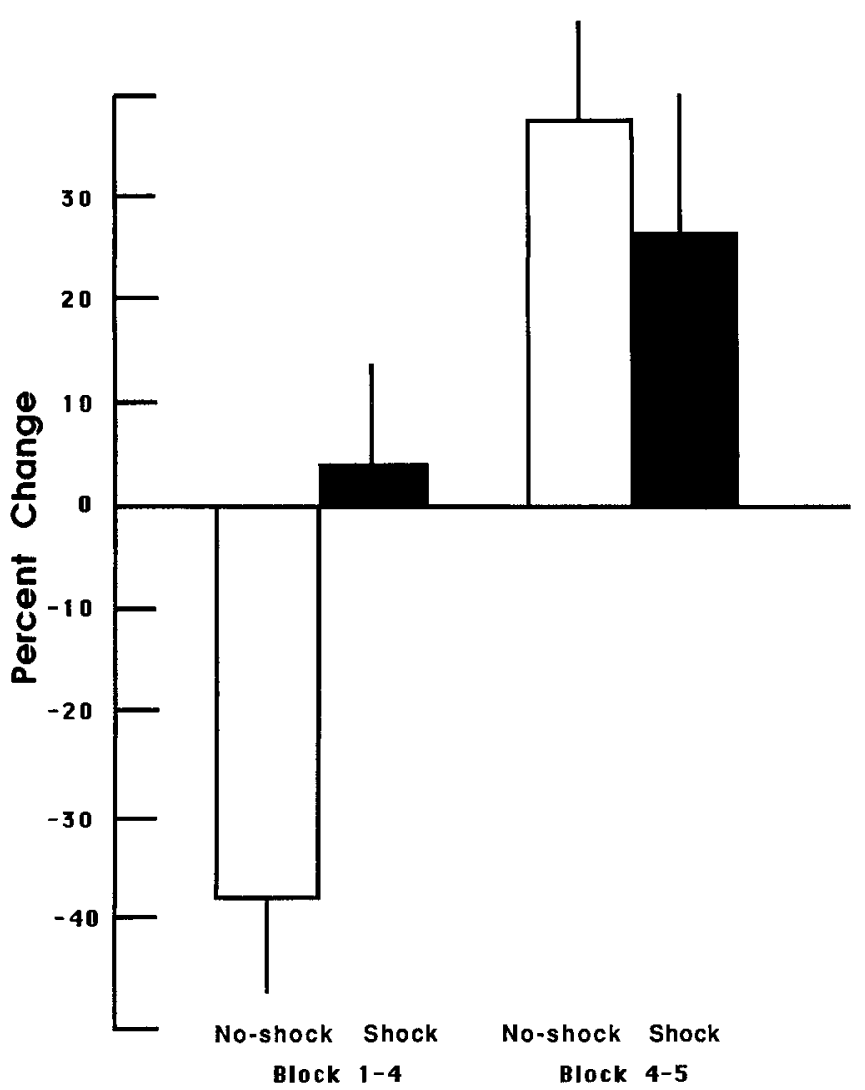

Figure 6. Mean percentage differences, Blocks 1 vs 4 and Blocks 4 vs 5 , for leeches in the no-shock (white bars) and shock (black bars) groups.

showed a significant decrement in response amplitude from Block 1 to Block 4. Contrary to our initial expectations, no difference in initial responding between the 2 groups was observed: $p>$ 0.1 . Neither were there differences in the absolute magnitude of the response or in the percentage of initial contraction. In a separate ANOVA on Blocks 4 and 5, leeches in both groups showed an increase in responding following the dishabituating stimulus block effect: $F(1,8)=11.27, p<0.01$; although leeches in the shock group showed significantly more responding overall; group effect: $F(1,8)=12.19, p<0.01$.

In order to further characterize the difference between the 2 groups, we calculated 2 difference scores for each leech, one from Blocks 1 to 4 and a second from the difference of responding from Blocks 4 to 5 . The difference scores are presented in Figure 6. A $t$ test for independent means indicated that the difference scores for Blocks 1 and 4 were significantly greater for leeches in the shock group than for those in the no-shock group: $t(8)=3.212, p<0.01$. In contrast, no differences were found in the Block 4 to Block 5 difference scores between leeches in the 2 groups: $t(8)=0.76, p>0.5$.

Experiment 3 demonstrates several important facts. First, the results from the NS group replicate the findings of Experiment 1. Second, a noxious stimulus presented prior to habituation did not cause an increase in baseline response, while it did retard subsequent habituation. The same noxious stimulus produced a significant increase in responding when presented to leeches in the shock group following habituation trials. In addition, this noxious stimulus resulted in a significant increase in responding back toward baseline for leeches in the no-shock group, which had shown significant habituation. Thus, a stimulus that pro- 
duces dishabituation of a decremented response does not produce sensitization, measured as an increased response magnitude. Across training, though, the noxious stimulus has a dramatic effect on the behavior; the presentation of the stimulus prior to habituation training dramatically retards the subsequent response decrement.

An alternative interpretation of the observed results is that the lack of an increase in responsiveness as a result of the sensitizing stimulus is simply due to a "ceiling effect." For several reasons we do not feel that this is the case. First, we do see a significant increase in responding in this same group following the presentation of the dishabituating stimulus. Second, in classical conditioning experiments, paired presentations of a CS (the tactile stimulus used in these experiments) with an unconditioned stimulus (the noxious stimulus used with these experiments) results in an enhancement of the response to the CS significantly above baseline responding (Sahley and Ready, 1985, 1988). Third, individual animals often exceed this "ceiling." Last, the habituating stimulus used is set only slightly above threshold in order to minimize stress. The animals in the study were therefore capable of responding at a level that exceeded the baseline used.

This pattern of results suggests that a noxious stimulus has at least 2 effects on this reflex: (1) it restores the reflex following habituation and (2) it prevents the habituation of a nondecremented response. Since the effect of the stimulus is dependent on its magnitude (Groves et al., 1969), we suggest that if a stronger stimulus were used, an increase in the magnitude of the reflex would be observed. Likewise, if a less intense stimulus were used, perhaps habituation would proceed in a way more similar to that observed for leeches in the control group. If this were the case, a third effect of a noxious stimulus would be an increase in responding over baseline (sensitization).

\section{General Discussion}

\section{Habituation and dishabituation}

The results of these 3 experiments clearly indicate that the touchelicited shortening response of the semi-intact leech preparation is modified by habituation, dishabituation, and sensitization. The observed plasticity of the semi-intact preparation is quite similar to that observed in the intact animal (Stoller and Sahley, 1985). Habituation of the response occurs following 20 presentations of a tactile stimulus applied to the anterior dorsal surface, and dishabituation occurs following the presentation of a noxious stimulus. Moreover, the degree of habituation is dependent on the interstimulus interval. That is, habituation training results in progressively more habituation as the ITI goes from 10 sec to $5 \mathrm{~min}$. In fact, no behavioral decrement occurs with a 10 sec ITI (see Figs. 3, 4).

Our findings support the idea that 2 independent processes, habituation and sensitization, interact in the final determination of a behavioral response. Moreover, we suggest that the characteristics of the stimulus, the frequency and intensity, are critical determinants of the observed behavior. Experiment $2 \mathrm{dem}$ onstrates that the frequency of the stimulus plays a large role in the behavioral outcome. Although Thompson et al. (1973) suggest that the intensity of the stimulus is an important determinant, it is more probable that, as proposed earlier by Groves et al. (1969), it is not any one variable but rather the interaction of stimulus intensity and frequency that is the most important factor in the behavioral response. This suggests that with a weaker stimulus, more rapid habituation would be observed in the
$10 \mathrm{sec}$ group and a more intense stimulus would lead to an increment in the behavioral response.

Finally, presentation of the noxious stimulus following habituation training increases the magnitude of the reflex. The difference scores show that identical amounts of facilitation result from the dishabituating stimulus, despite the absolute levels of habituation attained in the individual groups (see Fig. 6).

\section{Sensitization}

The results of Experiment 3 indicate that presentation of a noxious stimulus prior to habituation training prevents subsequent habituation, in contrast to the case of leeches in a control group not experiencing the noxious stimulus. The effect of the stimulus lasts at least $40 \mathrm{~min}$. These findings demonstrate several important facts. First, the no-shock group replicates the habituation of the reflex that we have previously seen produced by repeated stimulations (Experiment 1). Second, the noxious stimulus presented prior to habituation did not cause an increase in baseline responding, but did retard subsequent habituation. When presented a second time as the dishabituating stimulus, the same noxious stimulus produced an increase in responding for leeches in Group S. Likewise, the noxious stimulus resulted in a significant increase in responding back toward baseline for leeches in the no-shock group, which had shown significant habituation.

Prevention of habituation by a noxious stimulus has not been described in much detail. We think that the finding is important for 2 reasons. First, recent experiments on Aplysia have indicated that dishabituation and sensitization may be distinct from each other both at a behavioral level (Rankin and Carew, 1988) and at a cellular level (Gingrich and Byrne, 1985; Hochner et al., 1986). Prevention of habituation may represent a novel additional process and/or a variation of the processes known to mediate dishabituation or sensitization. The ultimate answer requires the cellular analysis of these behavioral phenomena. Our semi-intact preparation provides us with a tool for examining the similarities and/or differences between the behavioral phenomena of dishabituation and sensitization. It is clear from recent cellular experiments that a noxious stimulus can have multiple effects. That is, many cells within a behavioral circuit can be modified as a result of sensitization training (Frost and Kandel, 1984; Sahley, 1987; Frost et al., 1988) and/or several cellular changes can occur within a given neuron (Hochner et al., 1986). The resultant behavioral outcome is determined by the interaction and balance of all of these changes.

Moreover, we would like to suggest that prevention of habituation as a result of sensitization produced by the unconditioned stimulus (US) may be a critical first step in the formation of associations. This is not a new idea. It was first suggested that it applied on a behavioral level by Humphrey (1933) and more recently by Davis (1970). In addition, experiments on latent inhibition demonstrate that habituation of a stimulus prior to training impairs conditioning to the habituated stimulus (Lubow and Moore, 1959; Lubow, 1973). Moreover, on a cellular level, Hawkins et al. (1983) have demonstrated that, in Aplysia, classical conditioning is the result, to a great extent, of an enhancement of the heterosynaptic facilitation produced by a sensitizing stimulus. In this paper we have demonstrated that one effect of a sensitizing stimulus can be the prevention of habituation of the touch-elicited shortening reflex. It will be interesting to determine whether prevention of habituation is important in the classical conditioning modification of this same reflex. 


\section{References}

Byrne, J. H., V. F. Castellucci, and E. R. Kandel (1978) Contribution of individual mechanoreceptor neurons mediating defensive gill-withdrawal in Aplysia. J. Neurophysiol. 41: 418-431.

Carew, T. J., H. M. Pinsker, and E. R. Kandel (1972) Long term habituation of a defensive withdrawal reflex in Aplysia. Science 175: $451-454$.

Castellucci, V., and E. R. Kandel (1976) Presynaptic facilitation as a mechanism for behavioral sensitization in Aplysia. Science 94: 11761178.

Castellucci, V., H. Pinsker, I. Kupfermann, and E. R. Kandel (1970) Neuronal mechanisms of habituation and dishabituation of the gillwithdrawal reflex in Aplysia. Science 167: 1745-1748.

Davis, M. (1970) Effects of ISI length and variability on startle response habituation to different intensities of tone. Psychonom. Sci. 12: 337-338.

Debski, E. A., and W. O. Friesen (1985) Habituation of swimming activity in the medicinal leech. J. Exp. Biol. 116:169-188.

Domjan, M., and B. Burkhardt (1984) The Principles of Learning and Behavior, Brooks/Cole, Monterey, CA.

File, S. E. (1973) Inter-stimulus interval and the rate of behavioural habituation. Q. J. Exp. Psychol. 25: 360-367.

Frost, W. N., and E. R. Kandel (1984) Sensitizing stimuli reduce the effectiveness of the L 30 inhibitory interneurons on the siphon withdrawal reflex circuit of Aplysia. Soc. Neurosci. Abstr. 10:510.

Frost, W. N., G. A. Clark, and E. R. Kandel (1987) Parallel processing of short-term memory for sensitization in Aplysia: A candidate mechanism for increasing response duration and response specificity. $J$. Neurobiol. (in press).

Gee, W. (1913) The behavior of leeches with special reference to its modifiability. U. C. Pub. Zool. 2: 197-305.

Gingrich, K. J., and J. H. Byrne (1985) Simulation of synaptic depression, posttetanic potentiation and presynaptic facilitation of synaptic potentials from sensory neurons mediating gill-withdrawal reflex in Aplysia. J. Neurophysiol. 53: 652-669.

Groves, P. M., and R. F. Thompson (1970) Habituation: A dual process theory. Psychol. Rev. 77: 419-450.

Groves, P. M., D. Lee, and R. F. Thompson (1969) Effects of stimulus frequency and intensity on habituation and sensitization in the acute spinal cat. Physiol. Behav. 4: 383-388.

Hawkins, R. D., V. F. Castellucci, and E. R. Kandel (1981) Interneurons involved in mediation and modulation of the gill-withdrawal reflex in Aplysia. II. Identified neurons produce heterosynaptic facilitation contributing to behavioral sensitization. J. Neurophysiol. 45: 315-326.

Hawkins, R. D., T. W. Abrams, T. J. Carew, and E. R. Kandel (1983) A cellular mechanism of classical conditioning in Aplysia: Activitydependent amplification of presynaptic facilitation. Science 219:400405.

Hawkins, R. D., G. A. Clark, and E. R. Kandel (1987) Cell biological studies of learning in simple invertebrate and vertebrate systems. In Handbook of Physiology, Section 1: The Nervous System, V. Mountcastle and F. Plum, eds., pp. 25-83, American Physiological Society, Bethesda, MD.

Hochner, B., M. Klein, S. Schacher, and E. R. Kandel (1986) Additional component in the cellular mechanism of presynaptic facilitation contributes to behavioral dishabituation in Aplysia. Proc. Natl. Acad. Sci. USA 83: 8794-8798.
Humphrey, G. (1933) The Nature of Learning in its Relation to the Living System, pp. 165-179, Harcourt, Brace, and Co., New York.

Ison, J., and G. Hammond (1971) Modification of the startle reflex in the rat by changes in the auditory and visual environments. $J$. Comp. Physiol. Psychol. 75: 435-452.

Kaiser, F. (1954) Beitrage zur bewegung physiologie der hirudinean (A survey of the behavioral physiology of Hirudinea). Zool. Jahrbuech. 65: 59-90.

Kandel, E. R. (1976) Cellular Basis of Behavior: An Introduction to Behavioral Neurobiology, Freeman, San Francisco.

Kandel, E. R. (1977) Neuronal plasticity and the modification of behavior. In Handbook of Physiology. The Nervous System I. Section 1, Cellular Biology of Neurons, E. R. Kandel, ed., pp. 1137-1182, Williams and Wilkins, Baltimore, MD.

Kandel, E. R,, and J. H. Schwartz (1982) Molecular biology of an elementary form of learning: Modulation of transmitter release by cyclic AMP. Science 218: 433-443.

Keppel, G. (1973) Design and Analysis: A Researcher's Handbook, Prentice Hall, Englewood Cliffs, NJ.

Kristan, W. B., S. McGirr, and S. Simpson (1982) Behavioral and mechanosensory neuron responses to skin stimulation in leeches. J. Exp. Biol. 94: 97-119.

Lockery, S. R., and W. Kristan (1985) Neural correlates of habituation and sensitization in the leech. Soc. Neurosci. Abstr. 11: 794.

Lockery, S. R., J. N. P. Rawlins, and J. A. Gray (1985) Habituation of the shortening reflex in the medicinal leech. Behav. Neurosci. 99: 333-341.

Lubow, R. E. (1973) Latent inhibition. Psychol. Bull. 79: 398-407.

Lubow, R. E., and A. U. Moore (1959) Latent inhibition: The effect of nonreinforced preexposure to the conditioned stimulus. J. Comp. Physiol. Psychol. 52: 415-419.

Pinsker, H., I. Kupfermann, V. Castellucci, and E. R. Kandel (1970) Habituation and dishabituation of the gill-withdrawal reflex in Aplysia. Science 167: 1740-1742.

Pinsker, H., W. A. Hening, T. J. Carew, and E. R. Kandel (1973) Longterm sensitization of a defensive withdrawal reflex in Aplysia. Science 182: 1039-1042.

Rankin, C. H., and T. J. Carew (1988) Dishabituation and sensitization emerge as separate processes during development in Aplysia. J. Neurosci. 8: 197-211.

Ratner, S. C. (1972) Habituation and retention of habituation in the leech (Macrobdella decora). J. Comp. Physiol. Psychol. 81: 115-121.

Sahley, C. L. (1987) Sensitization training modifies sensory neurons in the leech. Soc. Neurosci. Abstr. 13: 615.

Sahley, C. L., and D. F. Ready (1985) Associative learning modifies two behaviors in the leech, Hirudo medicinalis. Soc. Neurosci. Abstr. 11: 367.

Sahley, C. L., and D. F. Ready (1988) Associative learning modifies two behaviors in the leech, Hirudo medicinalis. J. Neurosci. 8: 46124620 .

Stoller, D., and C. L. Sahley (1985) Habituation and sensitization of the shortening reflex in the leech, Hirudo medicinalis. Soc. Neurosci. Abstr. 11: 367 .

Thompson, R. F., P. M. Groves, T. J. Teyler, and R. A. Roemer (1973) A dual process theory of habituation: Theory and behavior. In $\mathrm{Ha}$ bituation, Vol. 1., H. V. S. Peeke and M. J. Herz, eds., pp. 239-269, Academic, New York. 